\title{
Tissue Pharmacology of Da-Cheng-Qi Decoction in Experimental Acute Pancreatitis in Rats
}

\author{
Xianlin Zhao, ${ }^{1}$ Yumei Zhang, ${ }^{1}$ Juan Li, ${ }^{1}$ Meihua Wan, ${ }^{1}$ Shifeng Zhu, ${ }^{1}$ Hui Guo, \\ Jin Xiang, ${ }^{1}$ Edwin C. Thrower, ${ }^{2}$ and Wenfu Tang ${ }^{1}$ \\ ${ }^{1}$ Department of Integrative Medicine, Sichuan Provincial Pancreatitis Center, West China Hospital, Sichuan University, \\ Chengdu 610041, China \\ ${ }^{2}$ Department of Internal Medicine, Section of Digestive Diseases, Veterans Affairs Connecticut Healthcare of West Haven, \\ Yale University School of Medicine, New Haven, CT 06520, USA
}

Correspondence should be addressed to Wenfu Tang; hxtangwenfu@126.com

Received 11 January 2015; Revised 16 May 2015; Accepted 10 June 2015

Academic Editor: Cory S. Harris

Copyright (C) 2015 Xianlin Zhao et al. This is an open access article distributed under the Creative Commons Attribution License, which permits unrestricted use, distribution, and reproduction in any medium, provided the original work is properly cited.

Objectives. The Chinese herbal medicine Da-Cheng-Qi Decoction (DCQD) can ameliorate the severity of acute pancreatitis (AP). However, the potential pharmacological mechanism remains unclear. This study explored the potential effective components and the pharmacokinetic characteristics of DCQD in target tissue in experimental acute pancreatitis in rats. Methods. Acute pancreatitislike symptoms were first induced in rats and then they were given different doses of DCQD $(6 \mathrm{~g} / \mathrm{kg}, 12 \mathrm{~g} / \mathrm{kg}$, and $24 \mathrm{~g} / \mathrm{kg}$ body weight) orally. Tissue drug concentration, tissue pathological score, and inflammatory mediators in pancreas, intestine, and lung tissues of rats were examined after 24 hours, respectively. Results. Major components of DCQD could be found in target tissues and their concentrations increased in conjunction with the intake dose of DCQD. The high-dose compounds showed maximal effect on altering levels of anti-inflammatory (interleukin-4 and interleukin-10) and proinflammatory markers (tumor necrosis factor $\alpha$ and interleukin-6) and ameliorating the pathological damage in target tissues $(P<0.05)$. Conclusions. DCQD could alleviate pancreatic, intestinal, and lung injury by altering levels of inflammatory cytokines in AP rats with tissue distribution of its components.

\section{Introduction}

Acute pancreatitis (AP) is an acute inflammation of the pancreas and surrounding tissue caused by pancreatic digestive enzymes. Although usually self-limiting, up to $20 \%$ of patients develop a severe form of disease, which can lead to a systemic inflammatory response syndrome (SIRS) and multiple organ dysfunction and failure [1]. It was reported that the overall mortality can range from 30 to $40 \%$ in the most severe cases [2,3], and many are related with multiple organ dysfunction and failure [4-6]. Intestinal injury caused by AP is closely associated with poor prognosis in severe AP patients and is believed to be the primary cause of acute lung injury progression [7-10]. Recently, the adjuvant use of herbal medicine and electroacupuncture ameliorated lung and intestinal injury and led to a marked reduction in morbidity and mortality in China [11-14].
Da-Cheng-Qi Decoction (DCQD) is composed of Dahuang (Radix et Rhizoma Rhei), Houpu (Magnolia officinalis REHD.), Zhishi (Fructus Aurantii Immaturus), and Mangxiao (Natrii Sulfas) and has been widely used to treat AP for over 30 years in China [13, 15-17]. Recent studies have shown that DCQD can promote gastrointestinal motility, reduce acute lung injury, and inhibit cytokine activity and inflammatory responses in AP [15-18]. However, more detailed insights into the molecular mechanism of DCQD are hampered by difficulties in identifying which specific components of the complex mixture produce the observed therapeutic effects [19-21]. The pharmacological characteristics and bioactivities of DCQD inside the target tissue of AP rats are still not clear, even though there has been some progress in serum pharmacology [19, 22-24].

In the current study, we evaluated the tissue distribution of DCQD and related pharmacodynamic effects in 
pancreatic, lung, and intestinal tissue following administration of different doses of DCQD in experimental AP in rats. Such findings will prove helpful in further understanding the mechanism of DCQD in treatment of AP.

\section{Materials and Methods}

2.1. Animals. Thirty male clean-grade, healthy SpragueDawley rats $(220 \pm 15 \mathrm{~g})$ were purchased from the Experimental Animal Center of West China Center of Medical Sciences of Sichuan University. All animal studies were fed, cared, and handled according to the Guide for the Care and Use of Laboratory Animals of Sichuan University and the Animal Ethics Committee Guidelines of the Animal Facility of the West China Hospital and China. They were acclimatized to the facilities for one week and fasted for 24 hours prior to the experiment.

2.2. Preparation of DCQD. The spray-dried drug powders of DCQD, Dahuang, Houpu, Zhishi, and Mangxiao, were purchased from Chengdu Green Herbal Pharmaceutical Co. Ltd. (Chengdu, China). Before being orally administered to rats, the spray-dried powder was mixed $(12: 24: 12: 9)$ by weight and reconstituted with sterile distilled water at different concentrations for the crude drug of $0.6 \mathrm{~g} / \mathrm{mL}$, $1.2 \mathrm{~g} / \mathrm{mL}$, and $2.4 \mathrm{~g} / \mathrm{mL}$.

2.3. Animal Models and Treatment with DCQD. The rats were randomly divided into normal group (NG), model group (MG), low-dose treatment group (LDG), median-dose treatment group (MDG), and high-dose treatment group (HDG). In this study, the rat model of AP was induced by retrograde perfusion into the biliopancreatic duct of $3.5 \%$ sodium taurocholate (Sigma, St. Louis, MO, USA) $(1 \mathrm{~mL} / \mathrm{kg}$ body weight) at a rate of $0.2 \mathrm{~mL} / \mathrm{min}$ with a microinfusion pump $[16,25]$. As a control, NG received the same procedure with saline $(\mathrm{NaCl} 0.9 \%)$ instead of sodium taurocholate. In DCQD-treated groups, the rats recovered from anesthesia and were administered intragastrically different concentrations of DCQD $(10 \mathrm{~mL} / \mathrm{kg})$ in LDG $(6 \mathrm{~g} / \mathrm{kg})$, MDG $(12 \mathrm{~g} / \mathrm{kg})$, and HDG $(24 \mathrm{~g} / \mathrm{kg}) 2 \mathrm{~h}$ after the operation, respectively. In the NG and MG, rats were given an equal volume of saline with the same procedure.

2.4. Measurement of Histopathology of Pancreatic, Lung, and Intestinal Tissues. After $24 \mathrm{~h}$ following the procedure, rats were sacrificed and their pancreatic, lung, and intestinal tissues samples were collected for pathological examination and other analyses [11, 16]. Briefly, these collected tissues were fixed in $10 \%$ neutral formalin and embedded in paraffin, which were then cut into $5 \mu \mathrm{m}$ thick sections and stained with hematoxylin and eosin (HE) according to standard protocols. All the histopathology specimens were reviewed and scored in a blinded fashion by two independent pathologists using a scoring system for the extent and severity of tissue injury (0-4, edema, neutrophil infiltration, necrosis, and hemorrhage, resp.) as previously described [26-28]. The total histopathology score is the mean of the combined scores for each parameter from both investigators.

2.5. Measurement of Inflammatory Cytokine Levels in Tissues of Pancreas, Lung, and Intestine. The level of inflammatory cytokines, tumor necrosis factor $\alpha$ (TNF- $\alpha)$, interleukin- 4 (IL-4), IL-10, and IL-6 in pancreas, and lung and intestine tissue samples were assessed using the Milliplex MAP Rat Cytokine/Chemokine magnetic bead immunoassay kit (Millipore Corporation, Billerica, MA) following the manufacturers' instructions $[29,30]$. Briefly, diluted tissues homogenate samples and multiple premixed microbeads were added to a plate and the plate was incubated with agitation on a shaker. Then, the appropriate antibody detection mix was added into each well after washing. Each antibody was specific to a single cytokine. The plate was read on a MAGPIX Luminex xMAP instrument (Luminex Corp, Austin, TX) and analyzed with MILLIPLEX Analysis software version 3 (Millipore Corporation, Billerica, MA).

2.6. Measurement of Drug Concentrations of DCQD in Tissues of Pancreas, Lung, and Intestine. After $24 \mathrm{~h}$ following the procedure, pancreas, lung, and intestine tissues samples were collected and homogenized (10\%) to measure the drug concentrations of DCQD. Similar to the analysis of serum pharmacokinetics of DCQD in our previous study, the concentrations of the 10 main components of DCQD (emodin, rhein, naringin, etc.), in pancreas, lung, and intestine tissue homogenates $(10 \%)$, were measured by high performance liquid chromatography-mass spectrometry (HPLC-MS) as described $[19,23,24]$.

Briefly, HPLC-MS system, consisting of a LC-10ADvp pump (Shimadzu), a SIL-HTc autosampler (Kyoto, Japan), and an API3000 triple-quadrupole LC-MS system (CA, USA), was controlled with Drug and Statistics analyst 1.4.2 software (Chinese Pharmacological Society, Beijing, China). Separation was performed on a C18 guard column $(5 \mu \mathrm{m}$, $4.0 \mathrm{~mm} \times 2.0 \mathrm{~mm}$, Phenomenex Inc., Torrance, CA, USA) and a YMC-Pack ODS-A C18 column $(5 \mu \mathrm{m}, 150 \mathrm{~mm} \times$ $4.6 \mathrm{~mm}$, YMC, Kyoto, Japan). The mobile phase consisted of methanol-water $(92: 8, \mathrm{v} / \mathrm{v})$ at a flow rate of $0.3 \mathrm{~mL} / \mathrm{min}$. The column was maintained at ambient temperature and the injection volume was $80 \mu \mathrm{L}$. A mass spectrometer was operated using an electrospray source configured to the negative ion mode and quantification was performed by multiple reaction monitoring (MRM) [23].

Ten calibration standards were prepared by spiking $200 \mu \mathrm{L}$ of blank tissue with $100 \mu \mathrm{L}$ of each working solution to obtain tissue concentration of $400,200,100,50,25$, $12.5,6.25$, and $3.13 \mathrm{ng} / \mathrm{mL}$ for emodin, 5000, 3750, 2500, $1250,625,312.5,156.25,78.13,39.06$, and $19.53 \mathrm{ng} / \mathrm{mL}$ for rhein, $160,120,80,40,20,10,5,2.5,1.25$, and $0.63 \mathrm{ng} / \mathrm{mL}$ for rheochrysidin, and $800,600,400,200,100,50,25$, $12.5,6.25$, and $3.13 \mathrm{ng} / \mathrm{mL}$ for aloe-emodin, chrysophanol, naringin, naringenin, hesperidin, magnolol, and honokiol. Quality control (QC) samples were prepared to obtain tissue concentrations of $3750,625,156.25$, and $39.06 \mathrm{ng} / \mathrm{mL}$ for rhein, 100, 25, and $6.25 \mathrm{ng} / \mathrm{mL}$ for emodin, 600, 100, 25, 
and $6.25 \mathrm{ng} / \mathrm{mL}$ for aloe-emodin, chrysophanol, naringin, naringenin, hesperidin, magnolol, and honokiol, and 120, 20,5 , and $1.25 \mathrm{ng} / \mathrm{mL}$ for rheochrysidin. The spiked samples (standard and QC samples) were pretreated and detected in each analytical batch along with the unknown samples [23].

Data collection, peak integration, and calibration were all calculated with Analyst 1.4.2 software. Calibration curves were plotted according to the peak area ratio of analytes to internal standards (ibuprofen), and the linear regression between tissue concentration and peak area ratio was determined by $1 / x^{2}$. Concentrations of QC and unknown samples were measured by interpolation from the calibration curves $[23,24]$.

2.7. Statistical Analysis. All data were expressed as mean \pm standard errors of mean (SEM). Statistical analysis was carried out using the PEMS3.1 statistical program. Oneway repeated-measures ANOVA (followed by multiple pairwise comparisons using Student-Neuman-Keuls procedure) was used for the analysis of differences among groups. For comparison, the level of statistical significance was set at $P<$ 0.05 or less.

\section{Results}

3.1. DCQD Alleviated Pathological Damage in Pancreatic, Lung, and Intestinal Tissue. In NG, the pancreas exhibited no sign of edema and necrosis. However, the MG showed the features of AP characterized by expansion of interstitial edema, extensive infiltration of inflammatory cells, obvious pancreatic acinar cell vacuolization, necrosis, and hemorrhage. The rats treated with DCQD had a significant reduction of inflammatory cell infiltration, hemorrhaging, necrosis, and interstitial edema compared to MG, the greatest effect being seen in the HDG. DCQD reduced the standard pathological scores of the pancreas affected by experimental AP, and the scores of MDG and HDG were significantly lower than that in the MG at 24 hours (Figures 1(a1) and 1(a2)). Similar results could be found in intestinal and lung tissue of animals with experimental AP (Figures 1(b1), 1(b2), 1(c1), and 1(c2)).

\subsection{DCQD Reduced Proinflammatory Cytokine Infiltration} and Increased Anti-Inflammatory Cytokine Expression in Pancreatic, Lung, and Intestinal Tissue. In the AP model group, there was a significant increase of inflammatory cytokine infiltration of pancreatic tissue at $24 \mathrm{~h}$ after sodium taurocholate stimulation compared with the sham operation group, especially the proinflammatory cytokine IL-6. In the DCQD-treated group, proinflammatory cytokine (IL-6 and TNF- $\alpha$ ) infiltration was significantly lower, and the antiinflammatory cytokine levels (IL-4 and IL-10) were significantly higher than that in the AP model group $(P<0.05)$ (Figure 2(a)). The greatest effect was seen in the HDG $(P<$ $0.01)$. Similar results were found in intestine and lung tissues of experimental AP (Figures 2(b) and 2(c)).

3.3. Major Components of DCQD Become Distributed in Pancreatic, Lung, and Intestinal Tissue. Concentrations of major components of DCQD in pancreatic, lung, and intestinal tissue of rats treated with DCQD were measured using a sensitive HPLC-TMS method. The result showed that the five major components of DCQD that were most abundant in the pancreatic tissue of rats after oral administration are naringenin, naringin, rhein, aloe-emodin, and emodin. The concentration of these components increased concurrently with the oral dose of DCQD other than naringenin (Figure 3(a)). Similar results could be found in intestinal and lung tissues (Figures 3(b) and 3(c)). The highest tissue component level of DCQG was rhein in pancreas, naringenin in intestine, and emodin in lung when given high-dose DCQD, respectively (Figure 3). It also was found that the concentration of the major components of DQCD in intestinal tissue was higher than that in pancreas and lung tissue.

\section{Discussion}

The biological effects of specific DCQD components and their tissue distribution have been a subject for much discussion. Previous studies have determined 4-10 major constituents of DCQD in dog and rat serum by HPLC-TMS method [19, 22, $23,31]$, but the targeting of specific tissues, such as pancreas, lung, and intestine, by these components remain unclear. This study showed that the most abundant components of DCQD in target tissue of rats had a similar distribution with that seen in serum after oral administration [19, 31]. However, the concentration of the major components of DCQD, such as naringenin, naringin, rhein, aloe-emodin, and emodin, in intestinal tissue was higher than that in pancreas and lung tissue. These findings indicate that major components of DCQD may be absorbed from the intestine and can end up in target tissue via the blood transport system. Our finding is consistent with the hypothesis of tissue pharmacology [32]. That is to say that the specific tissue pharmacology of components of Chinese medicine differs, depending on the target tissue; for example, the maximal levels of rhein were found in pancreas, emodin in lung, and naringenin in intestine, respectively. The blood-tissue barriers in different tissue may explain these phenomena. At present, most studies focus on the blood-brain barrier and gut barrier, whereas research regarding blood-pancreas barrier and blood-lung barrier is rare. The only study to touch upon this was from Burns et al. [33] who reported a role for the blood-pancreatic barrier in antibiotic excretion in chronic pancreatitis decades ago. Future research should focus on the blood-pancreatic barrier as this may play an important role in treatment of $\mathrm{AP}$, given that the pancreas is the origin of inflammatory cytokines $[6,34]$.

AP is a common and potentially lethal acute inflammatory disease, which is thought to be mediated by a variety of pro- and anti-inflammatory mediators released from the pancreas and various other sources during the course of the disease $[34,35]$. Local recruitment and activation of inflammatory cells in injured pancreas may lead to the production of proinflammatory cytokines, such as IL- 6 and TNF- $\alpha$, as well as anti-inflammatory IL-4 and IL-10. The imbalance of proinflammatory and anti-inflammatory cytokines could lead to the inflammatory cascade and injure intestine and lung tissue, 

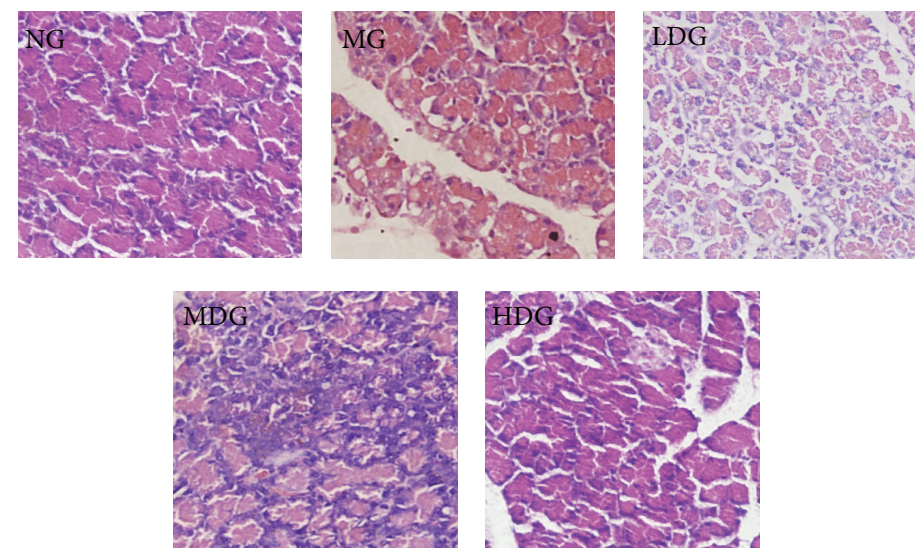

(a1)
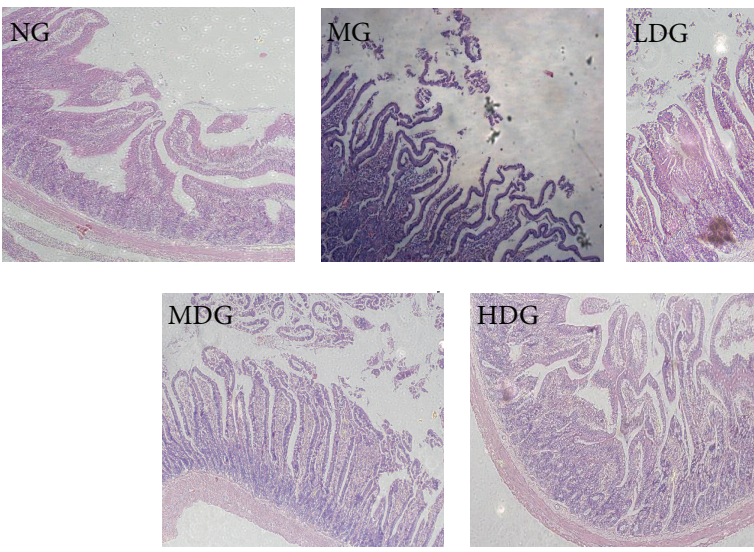

(b1)
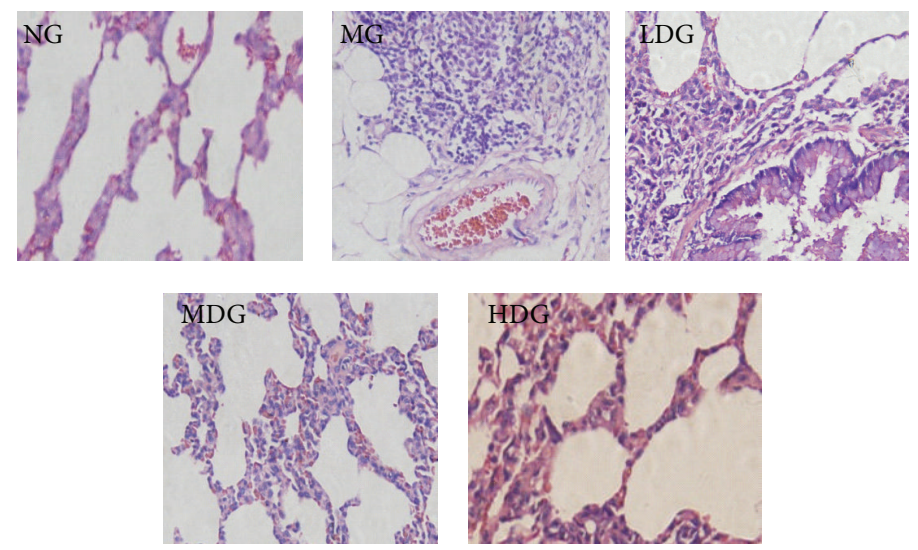

(c1)

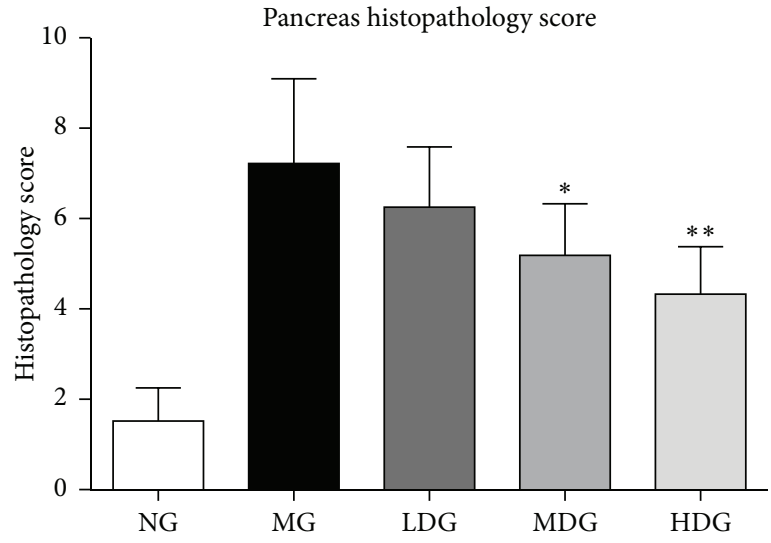

(a2)

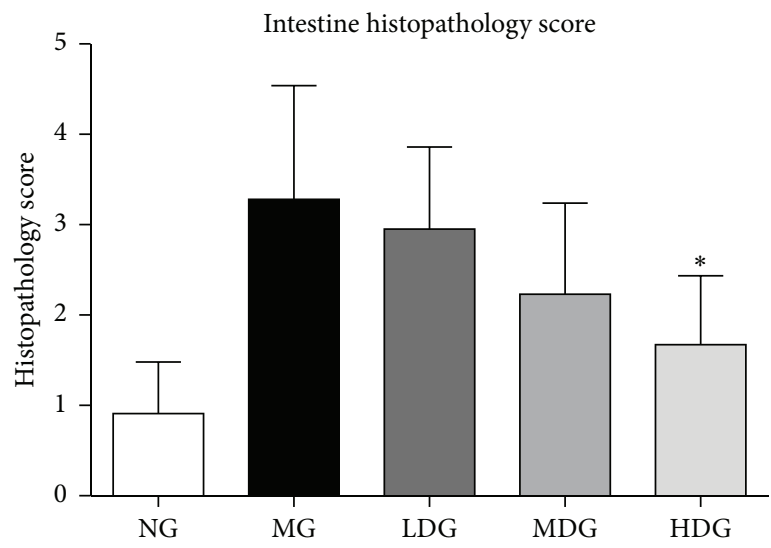

(b2)

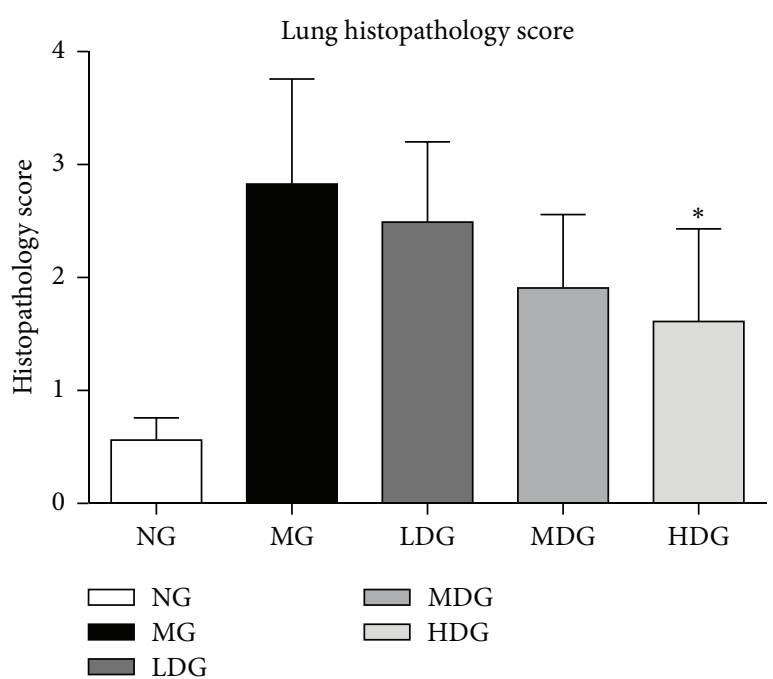

(c2)

FIGURE 1: DCQD alleviated acute pancreatitis-associated tissue damage. Normal group = NG, model group = MG, low-dose group = LDG, medium-dose group $=$ MDG, high-dose group $=$ HDG. Rats $(n=6$ per group $)$ were given different dose of DCQD $(6 \mathrm{~g} / \mathrm{kg}$ in LDG, $12 \mathrm{~g} / \mathrm{kg}$ in MDG, and $24 \mathrm{~g} / \mathrm{kg}$ in HDG by body weight) $2 \mathrm{~h}$ after operation. After $24 \mathrm{~h}$, the lung, intestine, and pancreas tissues were collected for pathological examination by hematoxylin and eosin (HE) staining. (a1) Pathological picture of pancreas (HE, $\times 400)$. (a2) Pathological scores of pancreas injury. (b1) Pathological picture of intestine (HE, $\times 100)$. (b2) Pathological scores of intestine injury. (c1) Pathological picture of lung $(\mathrm{HE}, \times 400)$. (c2) Pathological scores of lung injury. The results are mean \pm SE. ${ }^{*} P<0.05$ and ${ }^{* *} P<0.01$ versus AP model group. 


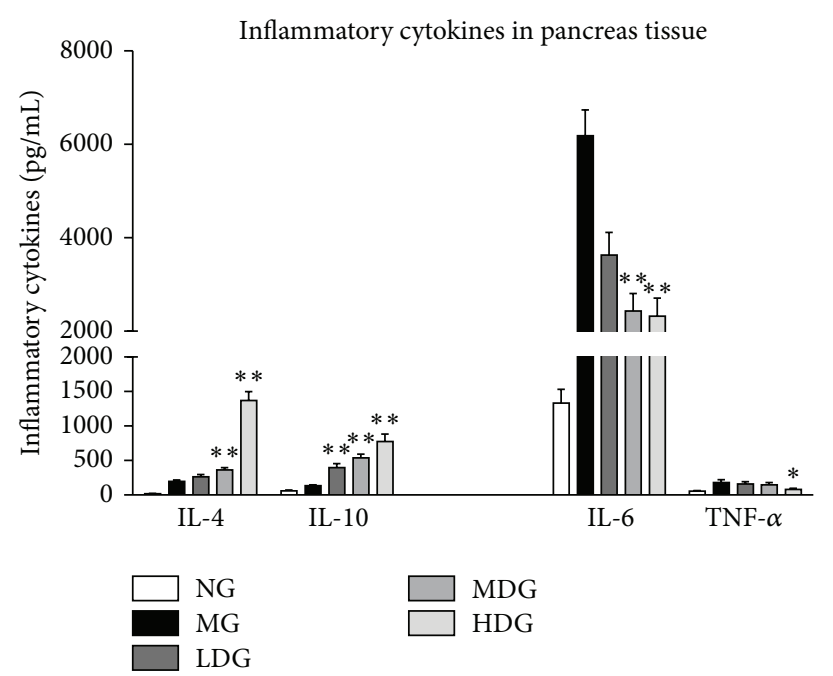

(a)

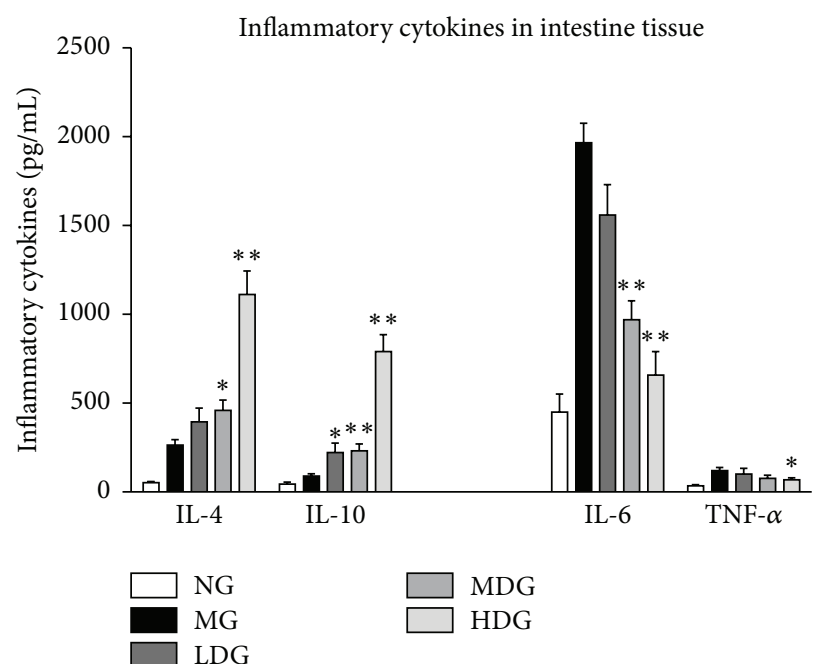

(b)

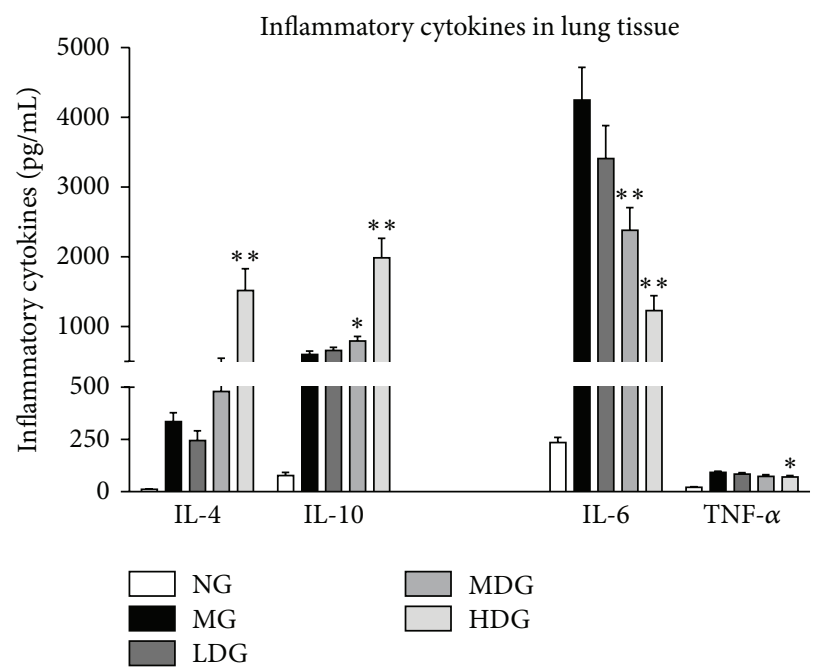

(c)

FIGURE 2: Effects of different dose of DCQD on the inflammatory cytokines in lung, intestine, and pancreas tissues. Normal group = NG, model group $=M G$, low-dose group $=\mathrm{LDG}$, medium-dose group $=\mathrm{MDG}$, high-dose group $=$ HDG. Rats $(n=6$ per group $)$ were given different dose of DCQD ( $6 \mathrm{~g} / \mathrm{kg}$ in LDG, $12 \mathrm{~g} / \mathrm{kg}$ in MDG, and $24 \mathrm{~g} / \mathrm{kg}$ in HDG by body weight) $2 \mathrm{~h}$ after operation. After $24 \mathrm{~h}$, the lung, intestine, and pancreas tissues were collected for examination of proinflammatory cytokines (IL- 6 and TNF- $\alpha$ ) and anti-inflammatory cytokines (IL- 4 and IL-10). (a) Inflammatory cytokines in pancreas tissues. (b) Inflammatory cytokines in intestine tissues. (c) Inflammatory cytokines in lung tissues. The results are mean $\pm \mathrm{SE} .{ }^{*} P<0.05$ and ${ }^{* *} P<0.01$ versus AP model group.

which aggravate the progression of AP $[10,36]$. In this study, we examined the value of IL-4, IL-6, IL-10, and TNF- $\alpha$ as predictors of inflammation in AP. Our result showed that the level of IL-6 was higher than other inflammatory cytokines and the level of TNF- $\alpha$ was lowest in pancreas, lung, and intestinal tissues at $24 \mathrm{~h}$. The role of proinflammatory IL6 in predicting severity of acute pancreatitis seems much more valuable than TNF- $\alpha$. DCQD treatment could increase the expression of anti-inflammatory cytokines (IL-4 and IL10) and inhibit the expression of proinflammatory cytokines (TNF- $\alpha$ and IL-6) to ameliorate the histopathological damage of acute pancreatitis, in which higher dose DCQD seems to have the better effect. These data support a DCQD-regulated systemic proinflammatory media/anti-inflammatory media balance in rats. Similar results can be found in other studies. Our previous studies found that rats treated with DCQD had lower mean pathological lung lesion scores, lower level of IL-6 mRNA, and higher expression of IL-10 mRNA than those in AP rats [18]. Huang et al. [12] reported that revised DCQD may inhibit IL-6 induction and increase IL-10 concentration and HSP70 expression, effectively reducing lung injury. It is important to note that some research studies reported that certain monomer components of DCQD can also exert antiinflammatory actions. For example, Gao et al. [37] reported that rhein could inhibit nuclear factor- $\kappa \mathrm{B}(\mathrm{NF}-\kappa \mathrm{B})$ activation and sequentially suppresses its downstream inducible nitric 


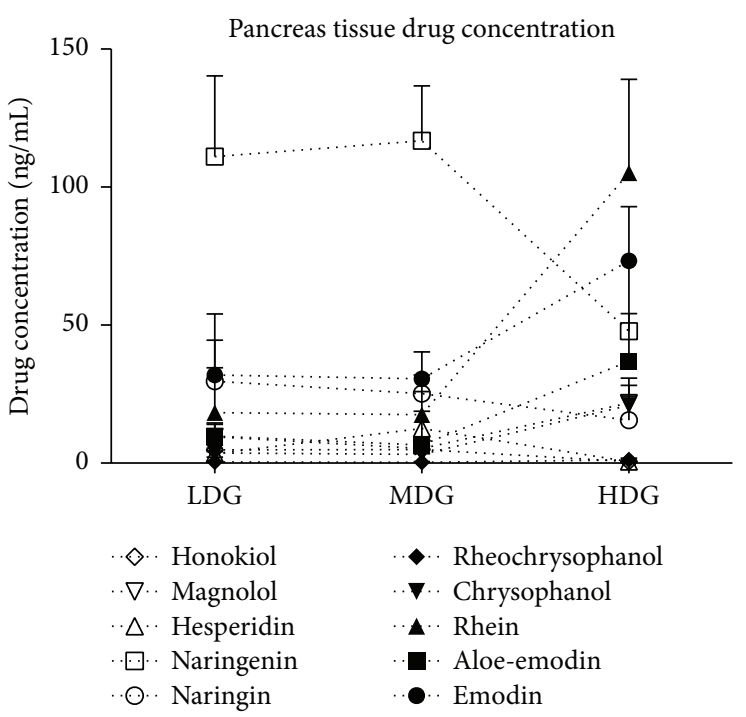

(a)

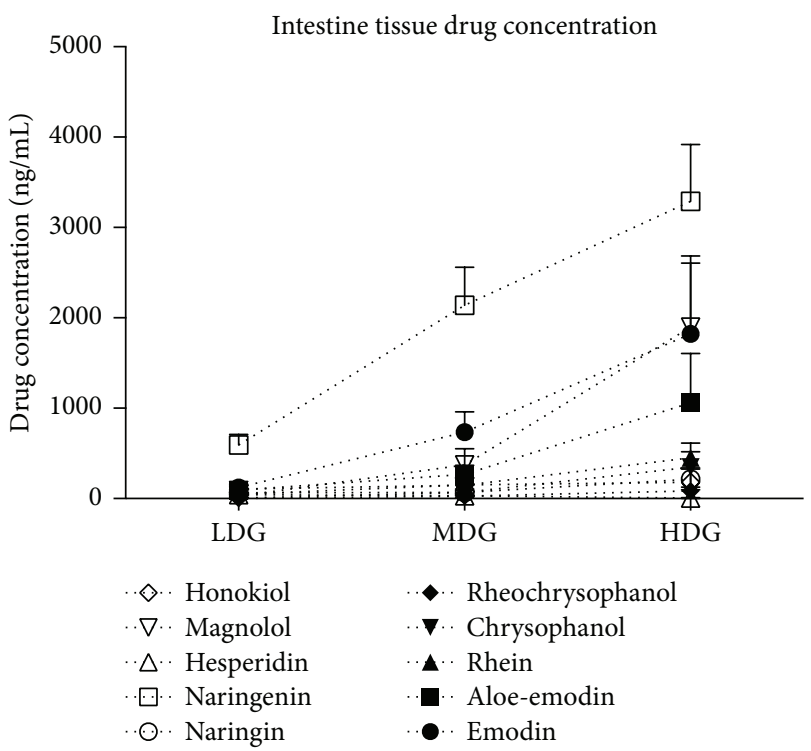

(b)

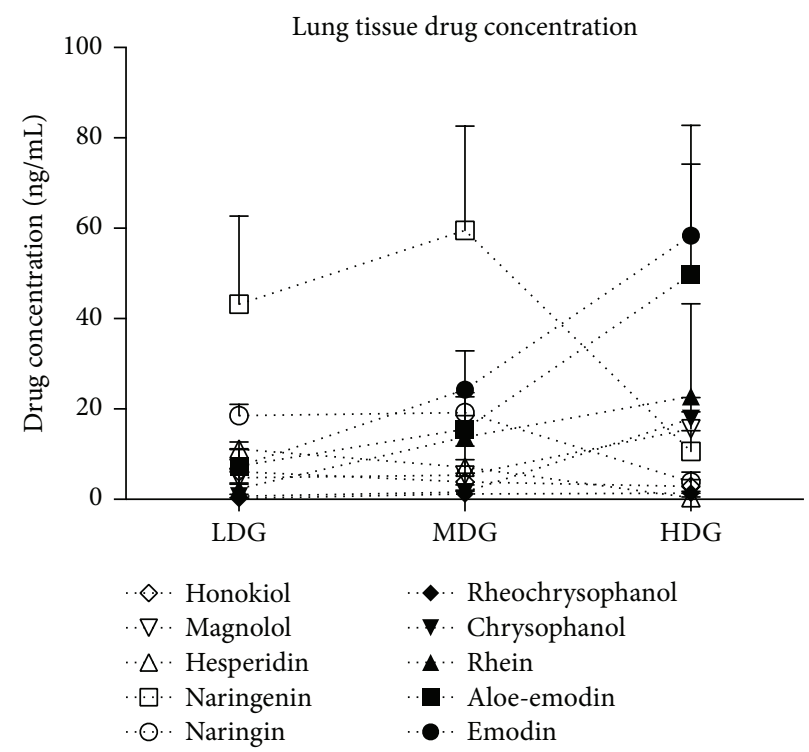

(c)

FIGURE 3: Distribution of different dose of DCQD in lung, intestine, and pancreas tissues. Normal group = NG, model group = MG, low-dose group $=$ LDG, medium-dose group $=$ MDG, high-dose group $=$ HDG. Rats $(n=6$ per group) were given different dose of DCQD $(6 \mathrm{~g} / \mathrm{kg}$ in LDG, $12 \mathrm{~g} / \mathrm{kg}$ in MDG, and $24 \mathrm{~g} / \mathrm{kg}$ in HDG by body weight) $2 \mathrm{~h}$ after operation. After $24 \mathrm{~h}$, the lung, intestine, and pancreas tissues were collected for examination of drug concentration of DCQD. (a) Drug concentration in pancreas tissues. (b) Drug concentration in intestine tissues. (c) Drug concentration in lung tissues. The results are mean \pm SE.

oxide synthase, IL-6, and TNF- $\alpha$ by inhibiting IKK $\beta$ in LPSactivated macrophages. Han et al. [38] reported that emodin treatment could attenuate IL-1 $\beta$ secretion via the inhibition of NOD-like receptor family, pyrin domain containing 3 (NLRP3) inflammasome activation in LPS-induced endotoxin mouse models. In view of the close relationship between DCQD and inflammatory cytokines, future research should focus on the link between quantified molecules of DCQD and their pharmacological effects with respect to their target tissue.
In summary, we confirmed that major components of DCQD could be absorbed into the target tissue of AP and ameliorate the histopathological damage by increasing the expression of the anti-inflammatory cytokines and inhibiting the expression of proinflammatory cytokines, which may be associated with the intake dose of DCQD. Further studies should investigate these ingredients in greater detail to take into account the specific molecular mechanisms in treatment of AP and help optimize herbal formulations as a therapy. 


\section{Conflict of Interests}

The authors declare that there is no conflict of interests regarding the publication of this paper.

\section{Authors' Contribution}

Xianlin Zhao and Yumei Zhang contributed equally to this paper. Wenfu Tang studied concept and design; Xianlin Zhao, Yumei Zhang, Juan Li, Hui Guo, Jin Xiang, and Shifeng Zhu were responsible for the acquisition of data; Xianlin Zhao, Yumei Zhang, Juan Li, and Meihua Wan were responsible for the analysis and interpretation of data; Xianlin Zhao and Yumei Zhang were responsible for drafting of the paper; Wenfu Tang and Edwin C. Thrower were responsible for the critical revision of the paper; Wenfu Tang obtained funding and studied supervision.

\section{Acknowledgment}

This study was supported by the National Natural Science Foundation of China (Grant no.: 81374042).

\section{References}

[1] J.-L. Frossard, M. L. Steer, and C. M. Pastor, "Acute pancreatitis," The Lancet, vol. 371, no. 9607, pp. 143-152, 2008.

[2] P. Banks, M. Freeman, R. Fass et al., "Practice guidelines in acute pancreatitis," The American Journal of Gastroenterology, vol. 101, no. 10, pp. 2379-2400, 2006.

[3] R. Flint, J. Windsor, and M. Bonham, "Trends in the management of severe acute pancreatitis: interventions and outcome," ANZ Journal of Surgery, vol. 74, no. 5, pp. 335-342, 2004.

[4] P. A. Banks, T. L. Bollen, C. Dervenis et al., "Classification of acute pancreatitis-2012: revision of the Atlanta classification and definitions by international consensus," Gut, vol. 62, no. 1, pp. 102-111, 2012.

[5] Y. Wang, W. Liu, X. Liu et al., "Role of liver in modulating the release of inflammatory cytokines involved in lung and multiple organ dysfunction in severe acute pancreatitis," Cell Biochemistry and Biophysics, vol. 71, no. 2, pp. 765-776, 2015.

[6] M. L. Malmstrøm, M. B. Hansen, A. M. Andersen et al., "Cytokines and organ failure in acute pancreatitis: inflammatory response in acute pancreatitis," Pancreas, vol. 41, no. 2, pp. 271-277, 2012.

[7] E. A. Deitch, "Gut lymph and lymphatics: a source of factors leading to organ injury and dysfunction," Annals of the New York Academy of Sciences, vol. 1207, no. 1, pp. E103-E111, 2010.

[8] M. J. Krzyzaniak, C. Y. Peterson, G. Cheadle et al., "Efferent vagal nerve stimulation attenuates acute lung injury following burn: the importance of the gut-lung axis," Surgery, vol. 150, no. 3, pp. 379-389, 2011.

[9] R. Tian, J.-T. Tan, R.-L. Wang, H. Xie, Y.-B. Qian, and K.-L. Yu, "The role of intestinal mucosa oxidative stress in gut barrier dysfunction of severe acute pancreatitis," European Review for Medical and Pharmacological Sciences, vol. 17, no. 3, pp. 349-355, 2013.

[10] M.-T. Zhou, C.-S. Chen, B.-C. Chen, Q.-Y. Zhang, and R. Andersson, "Acute lung injury and ARDS in acute pancreatitis: mechanisms and potential intervention," World Journal of Gastroenterology, vol. 16, no. 17, pp. 2094-2099, 2010.
[11] H. Guo, S.-F. Zhu, R.-R. Zhang, X.-L. Zhao, M.-H. Wan, and W.-F. Tang, "Electroacupuncture ameliorates acute lung injury through promoting gastrointestinal motility in rats with acute pancreatitis," Evidence-Based Complementary and Alternative Medicine, vol. 2014, Article ID 943596, 8 pages, 2014.

[12] L. Huang, M.-H. Wang, Z.-Y. Cheng et al., "Effects of ChaiQin-Cheng-Qi decoction on acute pancreatitis-associated lung injury in mice with acute necrotizing pancreatitis," Chinese Journal of Integrative Medicine, 2012.

[13] Q. Xia, Z. Huang, J. Jiang, G. Cheng, X. Yang, and W. Tang, "Yi-Huo-Qing-Xia method as the main therapy in integrated traditional Chinese and western medicine on severe acute pancreatitis: a report of 1161 cases," Chinese Journal of Integrated Traditional and Western Medicine in Intensive and Critical Care, vol. 13, no. 3, pp. 131-134, 2006.

[14] M.-H. Wan, J. Li, H.-L. Gong et al., "Clinical observation on the effect of dexamethasone and Chinese herbal decoction for purgation in severe acute pancreatitis patients," Chinese Journal of Integrative Medicine, vol. 17, no. 2, pp. 141-145, 2011.

[15] W. Huang, T. Jin, M. Javed et al., "Da-Cheng-Qi Decoction (DCQD) protects against pancreatic damage in murine experimental acute pancreatitis (AP)," Pancreatology, vol. 13, no. 2, p. e34, 2013.

[16] J. Wang, G. Chen, H. Gong, W. Huang, D. Long, and W. Tang, "Amelioration of experimental acute pancreatitis with Dachengqi Decoction via regulation of necrosis-apoptosis switch in the pancreatic acinar cell," PLoS ONE, vol. 7, no. 7, Article ID e40160, 2012.

[17] M.-H. Wan, J. Li, W. Huang et al., "Modified Da-ChengQi Decoction reduces intra-abdominal hypertension in severe acute pancreatitis: a pilot study," Chinese Medical Journal, vol. 125, no. 11, pp. 1941-1944, 2012.

[18] J. Zhao, J. Chen, W. Tang, L. Wan, W. Xiong, and L. Zhou, "Effect of Da-Cheng-Qi decoction on pancreatitis-associated lung injury in patients and anti-inflammatory responses in rat models," Pharmaceutical Biology, vol. 49, no. 10, pp. 1058-1064, 2011.

[19] J. Zhao, W. Tang, J. Wang, J. Xiang, H. Gong, and G. Chen, "Pharmacokinetic and pharmacodynamic studies of four major phytochemical components of da-cheng-qi decoction to treat acute pancreatitis," Journal of Pharmacological Sciences, vol. 122, no. 2, pp. 118-127, 2013.

[20] S. P. Li, J. Zhao, and B. Yang, "Strategies for quality control of Chinese medicines," Journal of Pharmaceutical and Biomedical Analysis, vol. 55, no. 4, pp. 802-809, 2011.

[21] X.-M. Liang, Y. Jin, Y.-P. Wang, G.-W. Jin, Q. Fu, and Y.-S. Xiao, "Qualitative and quantitative analysis in quality control of traditional Chinese medicines," Journal of Chromatography A, vol. 1216, no. 11, pp. 2033-2044, 2009.

[22] W.-F. Tang, Q. Yu, M.-H. Wan et al., "Simultaneous determination and pharmacokinetic studies of aloe emodin and chrysophanol in rats after oral administration of Da-ChengQi decoction by high-performance liquid chromatography," Biomedical Chromatography, vol. 21, no. 7, pp. 701-707, 2007.

[23] Q. Yu, J. Xiang, W. Tang, M. Liang, Y. Qin, and F. Nan, “Simultaneous determination of the 10 major components of Da-ChengQi decoction in dog plasma by liquid chromatography tandem mass spectrometry," Journal of Chromatography B, vol. 877, no. 22, pp. 2025-2031, 2009.

[24] H.-L. Gong, W.-F. Tang, Q. Yu et al., "Effect of severe acute pancreatitis on pharmacokinetics of Da-Cheng-Qi Decoction 
components," World Journal of Gastroenterology, vol. 15, no. 47, pp. 5992-5999, 2009.

[25] N. Franco-Pons, J. Casas, G. Fabriàs et al., "Fat necrosis generates proinflammatory halogenated lipids during acute pancreatitis," Annals of Surgery, vol. 257, no. 5, pp. 943-951, 2013.

[26] A. J. Rongione, A. M. Kusske, K. Kwan, S. W. Ashley, H. A. Reber, and D. W. McFadden, "Interleukin 10 reduces the severity of acute pancreatitis in rats," Gastroenterology, vol. 112, no. 3, pp. 960-967, 1997.

[27] J. A. Belperio, M. P. Keane, M. D. Burdick et al., "Critical role for CXCR2 and CXCR2 ligands during the pathogenesis of ventilator-induced lung injury," The Journal of Clinical Investigation, vol. 110, no. 11, pp. 1703-1716, 2002.

[28] S. Wirtz, C. Neufert, B. Weigmann, and M. F. Neurath, "Chemically induced mouse models of intestinal inflammation," Nature Protocols, vol. 2, no. 3, pp. 541-546, 2007.

[29] J. Amaral, J. W. Lee, J. Chou, M. M. Campos, and I. R. Rodríguez, "7-Ketocholesterol induces inflammation and angiogenesis in vivo: a novel rat model," PLoS ONE, vol. 8, no. 2, Article ID e56099, 2013.

[30] E. L. Burnham, J. M. McCord, S. Bose et al., "Protandim does not influence alveolar epithelial permeability or intrapulmonary oxidative stress in human subjects with alcohol use disorders," American Journal of Physiology-Lung Cellular and Molecular Physiology, vol. 302, no. 7, pp. L688-L699, 2012.

[31] F. Xu, Y. Liu, H. Dong, R. Song, and Z. Zhang, "Pharmacokinetic comparison in rats of six bioactive compounds between DaCheng-Qi decoction and its parent herbal medicines," Natural Product Communications, vol. 5, no. 5, pp. 795-800, 2010.

[32] W. Tang, M. Wan, and X. Huang, "Tissue pharmacology of recipe: a new hypothesis," Chinese Traditional and Herbal Drugs, vol. 36, no. 149, pp. 1-3, 2005.

[33] G. P. Burns, T. A. Stein, and L. S. Kabnick, "Blood-pancreatic juice barrier to antibiotic excretion," American Journal of Surgery, vol. 151, no. 2, pp. 205-208, 1986.

[34] R. Hoque, A. F. Malik, F. Gorelick, and W. Z. Mehal, "Sterile inflammatory response in acute pancreatitis," Pancreas, vol. 41, no. 3, pp. 353-357, 2012.

[35] E. Fisic, G. Poropat, L. Bilic-Zulle, V. Licul, S. Milic, and D. Stimac, "The role of IL-6, 8, and 10, sTNFr, CRP, and pancreatic elastase in the prediction of systemic complications in patients with acute pancreatitis," Gastroenterology Research and Practice, vol. 2013, Article ID 282645, 6 pages, 2013.

[36] J. Escobar, J. Pereda, A. Arduini et al., "Role of redox signaling, protein phosphatases and histone acetylation in the inflammatory cascade in acute pancreatitis: therapeutic implications," Inflammation \& Allergy: Drug Targets, vol. 9, no. 2, pp. 97-108, 2010.

[37] Y. Gao, X. Chen, L. Fang et al., "Rhein exerts pro- and antiinflammatory actions by targeting IKK $\beta$ inhibition in LPSactivated macrophages," Free Radical Biology and Medicine, vol. 72, pp. 104-112, 2014.

[38] J.-W. Han, D.-W. Shim, W.-Y. Shin et al., "Anti-inflammatory effect of emodin via attenuation of NLRP3 inflammasome activation," International Journal of Molecular Sciences, vol. 16, no. 4, pp. 8102-8109, 2015. 


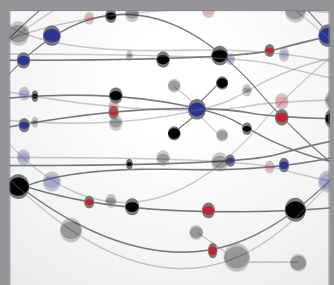

The Scientific World Journal
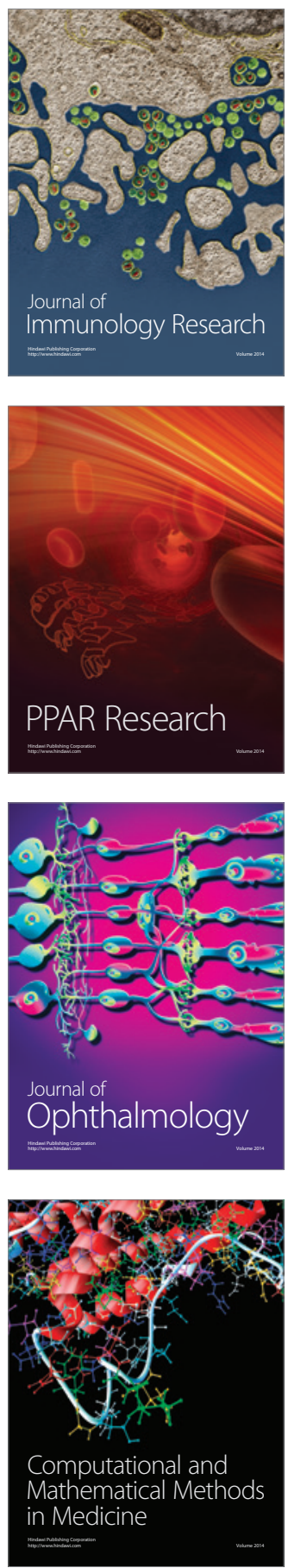

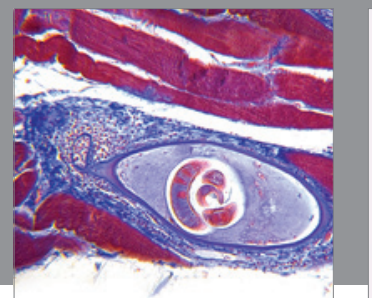

Gastroenterology

Research and Practice
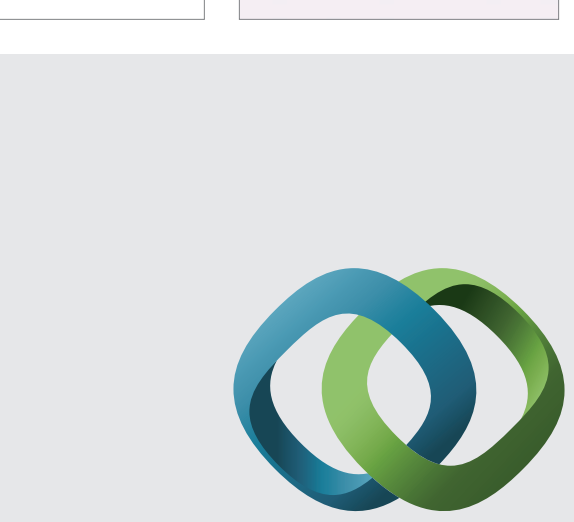

\section{Hindawi}

Submit your manuscripts at

http://www.hindawi.com
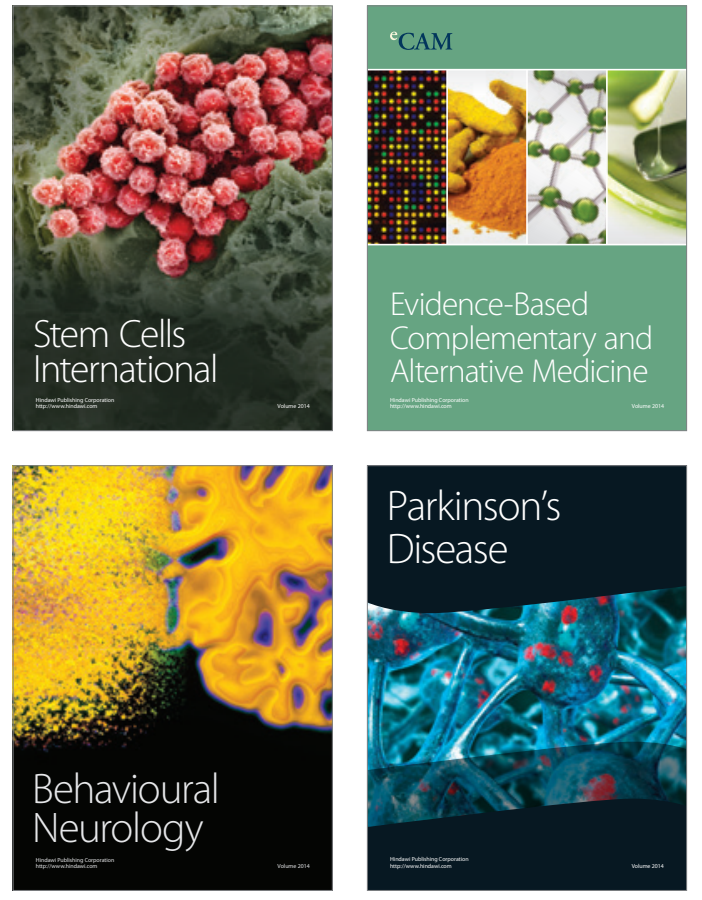
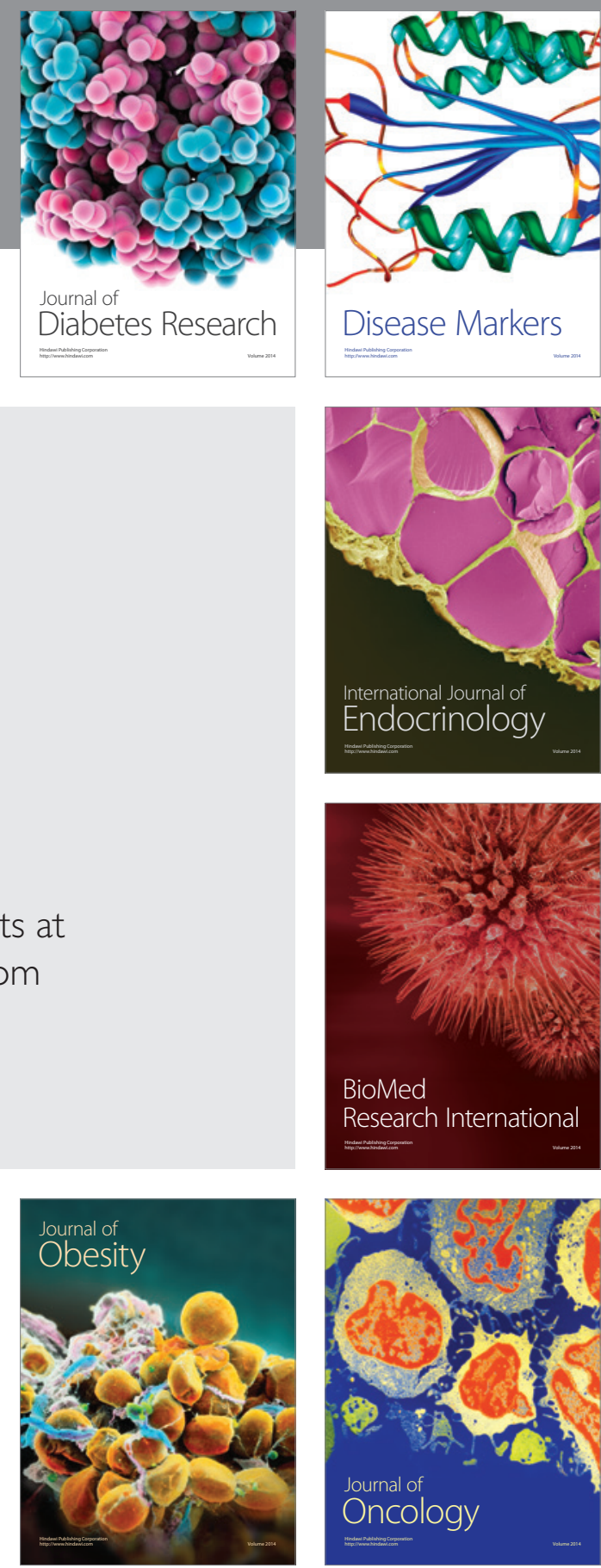

Disease Markers
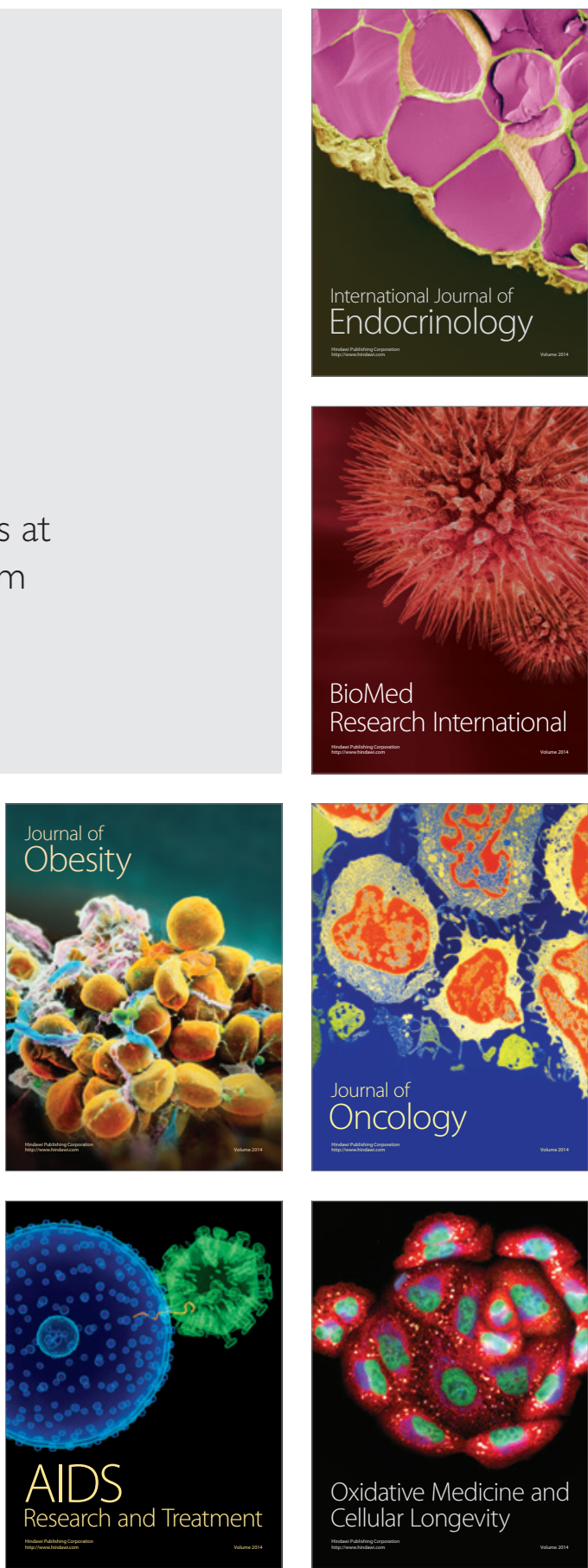